\title{
Heinz-Jürgen Schmidt*
}

\section{Geometry of the Rabi Problem and Duality of Loops}

https://doi.org/10.1515/zna-2019-0352

Received December 1, 2019; accepted January 24, 2020

Abstract: We investigate the motion of a classical spin processing around a periodic magnetic field using Floquet theory, as well as elementary differential geometry and considering a couple of examples. Under certain conditions, the role of spin and magnetic field can be interchanged, leading to the notion of "duality of loops" on the Bloch sphere.

Keywords: Floquet States; Periodically Driven Quantum Systems; Rabi Problem.

\section{Introduction}

The Rabi problem usually refers to the response of an atom to an applied harmonic electric field, with an applied frequency very close to the atom's natural frequency $[1,2]$. Assuming that the atom can be approximated by a twolevel system, its semiclassical Hamiltonian (in the sense that the radiation field is treated classically) will be of the form of a Zeeman term in an $s=1 / 2$ spin system:

$$
H=\omega_{0} S_{z}+b_{1}(t) S_{x}+b_{2}(t) S_{y}+b_{3}(t) S_{z},
$$

where the $S_{x}, S_{y}, S_{z}$ are the $s=1 / 2$ spin operators. If $\psi(t)$ is a solution of the corresponding Schrödinger equation $(\hbar=1)$ :

$$
i \frac{\mathrm{d}}{\mathrm{d} t} \psi(t)=H \psi(t)
$$

then the projector $P(t)=|\psi(t)\rangle\langle\psi(t)|$ can be expanded as a linear combination of the spin operators:

$$
P(t)=\frac{1}{2} \mathbb{1}+\mathbf{s}_{1}(t) S_{x}+\mathbf{s}_{2}(t) S_{y}+\mathbf{s}_{3}(t) S_{z} .
$$

It follows that $\mathbf{s}(t) \equiv\left(\mathbf{s}_{1}(t), \mathbf{s}_{2}(t), \mathbf{s}_{3}(t)\right)^{\top}$ will be a unit vector that obeys the same equation of motion

$$
\frac{\mathrm{d}}{\mathrm{d} t} \mathbf{s}(t)=\mathbf{h}(t) \times \mathbf{s}(t)
$$

\footnotetext{
*Corresponding author: Heinz-Jürgen Schmidt, Universität Osnabrück, Fachbereich Physik, D-49069 Osnabrück, Germany, E-mail: hschmidt@uos.de
}

as a classical magnetic moment performing a Larmor precession around the time-dependent periodic magnetic field $\mathbf{h}(t) \equiv\left(h_{1}(t), h_{2}(t), h_{3}(t)\right)^{\top} \equiv\left(b_{1}(t), b_{2}(t)\right.$, $\left.b_{3}(t)+\omega_{0}\right)^{\top}$. The study of this equation will be called the "classical Rabi problem" in what follows. Interestingly, the problem of a sphere rolling on a curved surface also leads to a differential equation of the form (4) [3, 4].

To illustrate the preceding remarks, consider the textbook example of the circularly polarised Rabi problem with

$$
\mathbf{h}(t)=\left(\begin{array}{c}
F \cos \omega t \\
F \sin \omega t \\
\omega_{0}
\end{array}\right) \text {. }
$$

A special solution of the corresponding Schrödinger equation (2) is the following:

$$
\begin{aligned}
\psi(t)= & \left(\begin{array}{l}
\psi_{1}(t) \\
\psi_{2}(t)
\end{array}\right) \\
= & \left(\begin{array}{c}
e^{-\frac{1}{2} i t \omega}\left(\cos \left(\frac{\Omega t}{2}\right)-\frac{i \Delta}{\Omega} \sin \left(\frac{\Omega t}{2}\right)\right) \\
-\frac{i F}{\Omega} e^{\frac{i \omega t}{2}} \sin \left(\frac{\Omega t}{2}\right)
\end{array}\right),
\end{aligned}
$$

where $\Delta$ is the "detuning"

$$
\Delta \equiv \omega_{0}-\omega
$$

and $\Omega$ denotes the "Rabi frequency"

$$
\Omega \equiv \sqrt{F^{2}+\Delta^{2}}
$$

This solution demonstrates the well-known Rabi oscillations of the occupation numbers of the eigenstates of the static Hamiltonian according to

$$
\left|\psi_{1}(t)\right|^{2}=1-\left|\psi_{2}(t)\right|^{2}=\frac{\Delta^{2}}{\Omega^{2}} \sin ^{2}\left(\frac{\Omega t}{2}\right)+\cos ^{2}\left(\frac{\Omega t}{2}\right) .
$$

However, the projector $P(t)$ defined in (3) has also components that are not $\frac{2 \pi}{\Omega}$-periodic, as is illustrated in Figure 1.

On the other hand, it can be shown $[5,6]$ that in the general case of periodic $\mathbf{h}(t)$ there will always exist 


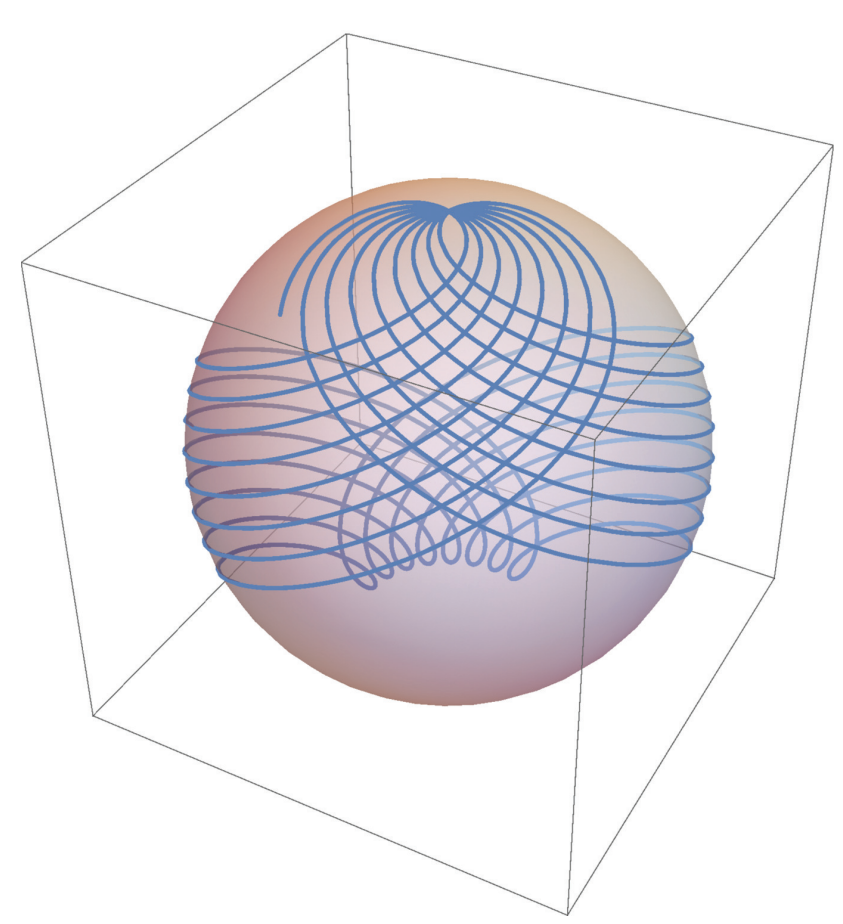

Figure 1: The curve swept by a classical spin vector $\mathbf{s}(t)$ that solves the equation of motion (4) with a circularly polarised magnetic field according to (5). The $z$-component of $\mathbf{s}(t)$ oscillates with the Rabi frequency $\Omega$. The physical parameters are chosen as $F=0.5, \omega=1$ and $\omega_{0}=0.9$.

$T \equiv \frac{2 \pi}{\omega}$-periodic solutions of (4) if the initial conditions are appropriately chosen. These solutions of the classical Rabi problem yield solutions of the underlying Schrödinger equation (2) up to a (time-depending) phase factor. But it turns out [5] that also this phase factor can be reconstructed from the periodic solution of (4) by means of certain integrals. Here we encounter the rare case where a quantum problem and the corresponding classical problem are essentially equivalent, due to the mathematical fact that the Lie groups $\mathrm{SO}(3)$ and $\mathrm{SU}(2)$ are locally isomorphic. This endows the classical Rabi problem with additional importance concerning quantum applications. Moreover, there are connections between quantum search Hamiltonians and exactly solvable time-dependent twolevel quantum systems [7].

We mention in passing that solutions of the classical Rabi problem also yield solutions of the quantum Rabi problem for arbitrary spin quantum number $s$. This follows either from representation theory [5] or by using the Majorana stellar representation [8] of spin states by $2 s$ points of the Bloch sphere. A third way to look at this is to consider spin fluctuation tensors, see, e.g. [9].

The differential equation (4) can be explicitly solved only in a few cases of physical interest. The most prominent one, already mentioned above, is a constant field superimposed by a monochromatical, circularly polarised field perpendicular to the constant one [1]. The analogous problem with a linearly polarised field component is solvable in terms of confluent Heun functions [10-13] for the corresponding $s=1 / 2$ Schrödinger equation. In this article, we will shift the problem of finding solutions of (2) or (4) to the study of geometric relations between such solutions and to the interplay between Floquet theory, differential geometry of the unit sphere, and duality of loops. Not all results will be new, but we will provide new proofs that only use properties of solutions of the classical Rabi problem that are easier to visualise and do not resort to the mathematics of the underlying Schrödinger equation. Obviously, there exist close connections between the present article and the theory of geometric phases, initiated by M. Berry [14], generalised by [15], and still a topic of current experimental research (e.g. [16-19]). However, a detailed account of these connections cannot be given here and must be left for future publications.

\section{Periodic Solutions}

We will sketch the essential arguments leading to periodic solutions of the classical Rabi problem and the reconstruction of the (time-depending) phase factor. First, we may apply the Floquet theory $[20,21]$ to the Schrödinger equation (2) and conclude that it has special solutions ("Floquet solutions") of the form

$$
\psi_{ \pm}(t)=u_{ \pm}(t) e^{-i \epsilon_{ \pm} t},
$$

such that $u_{ \pm}(t)$ will be $T$-periodic and the "quasienergies" $\epsilon_{ \pm}$are real numbers uniquely determined up to integer multiples of $\omega$. It can be shown that there exist representatives of quasienergies satisfying

$$
\epsilon_{ \pm}= \pm \epsilon \text { such that } \epsilon \geq 0
$$

and these will be chosen in the sequel. It follows immediately that the projectors $P_{ \pm}(t)=\left|\psi_{ \pm}(t)\right\rangle\left\langle\psi_{ \pm}(t)\right|$ will be $T$-periodic functions of $t$.

Conversely, let a $T$-periodic solution of (4) be given and $P(t)$ be the corresponding time-dependent projector defined by (3). It may be written as $P(t)=|\phi(t)\rangle\langle\phi(t)|, \phi(t)$ being $T$-periodic, such that the solution $\psi(t)$ of (2) we are looking for is of the form

$$
\psi(t)=e^{-i \alpha(t)} \phi(t)
$$


It follows that

$$
\begin{aligned}
0 & =\left(H-i \frac{\partial}{\partial t}\right) \psi(t) \\
& =e^{-i \alpha(t)}\left(H-i \frac{\partial}{\partial t}\right) \phi(t)-\frac{\mathrm{d} \alpha}{\mathrm{d} t} e^{-i \alpha(t)} \phi(t),
\end{aligned}
$$

and hence

$$
\begin{aligned}
\frac{\mathrm{d} \alpha}{\mathrm{d} t} & =\left\langle\phi(t)\left|H-i \frac{\partial}{\partial t}\right| \phi(t)\right\rangle \\
& =\sum_{n \in \mathbb{Z}} a_{n} e^{i n \omega t},
\end{aligned}
$$

where (16) represents the Fourier series of the $T$-periodic function (15). The integration of (16) yields

$$
\alpha(t)=a_{0} t+\sum_{n \neq 0} \frac{a_{n}}{i n \omega} e^{i n \omega t}
$$

and hence, $\psi(t)$ will be a Floquet solution of the form

$$
\psi(t)=\phi(t) \exp \left(-i \sum_{n \neq 0} \frac{a_{n}}{i n \omega} e^{i n \omega t}\right) e^{-i a_{0} t},
$$

with quasienergy $\epsilon=a_{0}$. It is plausible that $a_{0}$ can be expressed by an integral of a function of the components of $P(t)=|\phi(t)\rangle\langle\phi(t)|$ over one period (see [5] for the details). In the next section, we will derive an alternative integral representation of the quasienergy.

It will be instructive to consider another argument leading to a periodic solution of (4). To this end, we consider a $3 \times 3$ matrix $R(t)$, the columns of which are three solutions $\mathbf{s}^{(i)}(t), i=1,2,3$, of (4) such that the initial conditions can be written as

$$
R(0)=\mathbb{1},
$$

the latter denoting the $3 \times 3$ unit matrix. As the equation of motion (4) leaves scalar products invariant, it follows that the columns of $R(t)$ will always form a right-handed orthonormal frame, in other words, that $R(t) \in S O(3)$. The three equations of motion (4) for the $\mathbf{s}^{(i)}(t), i=1,2,3$ can be compactly written in matrix form as

$$
\frac{\mathrm{d}}{\mathrm{d} t} R(t)=H(t) R(t)
$$

where $H(t)$ is the real, antisymmetric $3 \times 3$ matrix

$$
H(t)=\left(\begin{array}{ccc}
0 & -h_{3}(t) & h_{2}(t) \\
h_{3}(t) & 0 & -h_{1}(t) \\
-h_{2}(t) & h_{1}(t) & 0
\end{array}\right),
$$

and the $h_{i}(t)$ are the components of the magnetic field according to (4). The value of $R(t)$ value after one period will be the rotational matrix $R(T) \in S O(3)$. As any rotational matrix with unit determinant, it will be a rotation about an axis with an angle $\delta$. Accordingly, $R(T)$ will have the eigenvalues $1, e^{ \pm i \delta}$, corresponding to a real eigenvector a and two generally complex eigenvectors, respectively. The eigenvector a satisfying

$$
R(T) \mathbf{a}=\mathbf{a}
$$

represents the axis of rotation and, after normalisation $\|\mathbf{a}\|=1$, will be unique up to a sign. Let $\mathbf{s}(t)=R(t) \mathbf{a}$ be the solution of (4) with initial value $\mathbf{s}(0)=\mathbf{a}$, then it follows that

$$
\mathbf{s}(T)=R(T) \mathbf{a}=\mathbf{a}=\mathbf{s}(0)
$$

and hence, $\mathbf{s}(t)$ is, up to a possible sign, the $T$-periodic solution of (4) that we have constructed above by considering a Floquet solution of (2).

The angle of rotation $\delta \geq 0$ corresponding to $R(T)$ is related to the quasienergy as follows:

\section{Proposition 1:}

$$
\delta=2 \epsilon T
$$

Proof. This follows from [5] by the "lift" from $s=1 / 2$ to $s=1$. Independently, one may directly prove the proposition by considering the unitary matrix of Floquet solutions

$$
U(t)=\left(\begin{array}{cc}
u(t) e^{-i \epsilon t} & -\overline{v(t)} e^{i \epsilon t} \\
v(t) e^{-i \epsilon t} & \overline{u(t)} e^{i \epsilon t}
\end{array}\right),
$$

and the superposition

$$
\widetilde{\psi}(t)=\frac{1}{\sqrt{2}} U\left(\begin{array}{l}
1 \\
1
\end{array}\right) .
$$

Here

$$
u(t)=u_{1}(t)+i u_{2}(t)
$$

and

$$
v(t)=v_{1}(t)+i v_{2}(t)
$$

are $T$-periodic functions satisfying

$$
|u(t)|^{2}+|v(t)|^{2}=1
$$


for all $t \in \mathbb{R}$. The corresponding projector $\widetilde{P}(t)=$ $|\widetilde{\psi}(t)\rangle\langle\widetilde{\psi}(t)|$ yields a solution $\widetilde{\mathbf{s}}(t)$ of (4) via (3), which is always orthogonal to the periodic solution $\mathbf{s}(t)$ according to (23). After some straightforward calculations, we obtain

$$
\begin{aligned}
\widetilde{P}_{11}(0) & =\frac{1}{2}\left(\left(u_{1}(0)-v_{1}(0)\right)^{2}+\left(u_{2}(0)+v_{2}(0)\right)^{2}\right) \\
\widetilde{P}_{12}(0) & =\widetilde{P}_{21}(0) \\
& =\frac{1}{2}\left(\left(u_{1}(0)+i u_{2}(0)\right)^{2}-\left(v_{1}(0)-i v_{2}(0)\right)^{2}\right) \\
\widetilde{P}_{22}(0) & =\frac{1}{2}\left(\left(u_{1}(0)+v_{1}(0)\right)^{2}+\left(u_{2}(0)-v_{2}(0)\right)^{2}\right)
\end{aligned}
$$

and

$$
\begin{aligned}
\widetilde{P}_{11}(T)= & \frac{1}{2}-\left(u_{2}(0) v_{1}(0)+u_{1}(0) v_{2}(0)\right) \sin (2 T \epsilon) \\
& +\left(u_{2}(0) v_{2}(0)-u_{1}(0) v_{1}(0)\right) \cos (2 T \epsilon) \\
\widetilde{P}_{12}(T)= & \overline{P_{21}(T)}=\frac{1}{2} e^{-2 i T \epsilon} \\
& \left(\left(u_{1}(0)+i u_{2}(0)\right)^{2}-\left(v_{1}(0)-i v_{2}(0)\right)^{2} e^{4 i T \epsilon}\right) \\
\widetilde{P}_{22}(T)= & \frac{1}{2}+\left(u_{2}(0) v_{1}(0)+u_{1}(0) v_{2}(0)\right) \sin (2 T \epsilon) \\
& +\left(u_{1}(0) v_{1}(0)-u_{2}(0) v_{2}(0)\right) \cos (2 T \epsilon)
\end{aligned}
$$
fies

Hence, $\widetilde{\mathbf{s}}(t)$ will in general not be $T$-periodic but satis-

$$
\operatorname{Tr}(\widetilde{P}(0) \widetilde{P}(T))=\frac{1}{2}(1+\widetilde{\mathbf{s}}(0) \cdot \widetilde{\mathbf{s}}(T))=\frac{1}{2}(1+\cos 2 \epsilon T),
$$

as can be shown by a straightforward calculation. This completes the proof of Proposition 1.

In view of Proposition 1, we will define the "classical quasienergy" by

$$
\epsilon^{(c l)} \equiv \frac{\delta}{T} \stackrel{(24)}{=} 2 \epsilon .
$$

\section{Quasienergy}

It has been shown [5] that the quasienergy of the $s=1 / 2$ Schrödinger equation (2) with a periodic magnetic field can be expressed in terms of integrals using the periodic solution of the analogous classical Rabi problem. Here we will rederive this result without employing the reference to the Schrödinger equation, solely by using the periodic solution $\mathbf{s}(t)$ of (4) considered in Section 2.

To this end, we consider a time-dependent righthanded orthonormal frame, shortly called "e frame," defined by

$$
\begin{gathered}
\mathbf{e}^{(1)}(t) \equiv \mathbf{s}(t), \\
\mathbf{e}^{(2)}(t) \equiv \frac{\dot{\mathbf{s}}(t)}{\|\dot{\mathbf{s}}(t)\|}, \\
\mathbf{e}^{(3)}(t) \equiv \mathbf{e}^{(1)}(t) \times \mathbf{e}^{(2)}(t) .
\end{gathered}
$$

Further, let

$$
S(t)=\left(\mathbf{s}^{(1)}(t), \mathbf{s}^{(2)}(t), \mathbf{s}^{(3)}(t)\right) \in S O(3)
$$

be a solution of (20) with initial conditions

$$
\mathbf{s}^{(i)}(0)=\mathbf{e}^{(i)}(0), \quad i=1,2,3,
$$

and hence

$$
\mathbf{s}^{(1)}(t)=\mathbf{e}^{(1)}(t)=\mathbf{s}(t)
$$

for all $t \in \mathbb{R}$. It follows that the other two components of $S(t)$ can be expanded with respect to the e frame in the form

$$
\begin{gathered}
\mathbf{s}^{(2)}(t)=\cos (\alpha(t)) \mathbf{e}^{(2)}(t)+\sin (\alpha(t)) \mathbf{e}^{(3)}(t), \\
\mathbf{s}^{(3)}(t)=-\sin (\alpha(t)) \mathbf{e}^{(2)}(t)+\cos (\alpha(t)) \mathbf{e}^{(3)}(t),
\end{gathered}
$$

where $\alpha(t)$ is a smooth function. The differential equation (20) yields a corresponding one for $\alpha(t)$ that can be solved in terms of an indefinite integral, see Appendix A. Recall that $\delta$ is the angle between $\mathbf{e}^{(2)}(T)=\mathbf{s}^{(2)}(0)$ and $\mathbf{s}^{(2)}(T)$. Hence, using the integral representation (113) of $\alpha(t)$, the classical quasienergy can be written as

$$
\epsilon^{(c l)} \stackrel{(37)}{=} \frac{\delta}{T}=\frac{1}{T} \int_{0}^{T}\left((\mathbf{h} \cdot \mathbf{s})-\frac{\mathbf{s} \cdot(\dot{\mathbf{s}} \times \ddot{\mathbf{s}})}{\dot{\mathbf{s}} \cdot \dot{\mathbf{s}}}\right) \mathrm{d} t .
$$

This result slightly improves the corresponding equation (62) in [5] in so far as it is manifestly independent of a coordinate system. The explicit accordance with [5] has been shown in [6]. We note that the form of (46) suggests the following splitting of the quasienergy:

$$
\begin{aligned}
\epsilon^{(c l)} & =\epsilon_{d}^{(c l)}+\epsilon_{g}^{(c l)} \\
& \equiv \frac{1}{T} \int_{0}^{T} \mathbf{h} \cdot \mathbf{s} \mathrm{d} t+\frac{1}{T} \int_{0}^{T}\left(-\frac{\mathbf{s} \cdot(\dot{\mathbf{s}} \times \ddot{\mathbf{s}})}{\dot{\mathbf{s}} \cdot \dot{\mathbf{s}}}\right) \mathrm{d} t
\end{aligned}
$$


into a "dynamical part" $\epsilon_{d}^{(c l)}$ and a "geometrical part" $\epsilon_{g}^{(c l)}$. The dynamical part $\epsilon_{d}^{(c l)}$ is obviously the time average of the energy $\mathbf{h} \cdot \mathbf{s}$ and depends on the details of the dynamics, not only on the curve $\mathcal{S}$ swept by the periodic solution $\mathbf{s}(t)$.

For the geometrical part $\epsilon_{d}^{(c l)}$, we note that the corresponding integral $\int_{0}^{T}(\ldots) \mathrm{d} t$ is invariant under an arbitrary parameter transformation $t \mapsto s(t)$ that leads to a new period $S$ :

$$
\begin{aligned}
& \int_{0}^{T}\left(-\frac{\mathbf{s} \cdot(\dot{\mathbf{s}} \times \ddot{\mathbf{s}})}{\dot{\mathbf{s}} \cdot \dot{\mathbf{s}}}\right) \mathrm{d} t \\
& =\int_{0}^{S}\left(-\frac{\mathbf{s} \cdot\left(\mathbf{s}^{\prime} \times \mathbf{s}^{\prime \prime}\right)}{\mathbf{s}^{\prime} \cdot \mathbf{s}^{\prime}}\right) \mathrm{d} s,
\end{aligned}
$$

where we have denoted the $s$ derivative by a prime'. This transformation produces a factor $\left(\frac{\mathrm{d} s}{\mathrm{~d} t}\right)^{3}$ in the numerator of the integrand of the left-hand side of (48) and a factor $\left(\frac{\mathrm{d} s}{\mathrm{~d} t}\right)^{2}$ in the denominator; after cancelling the remaining factor, $\frac{\mathrm{d} s}{\mathrm{~d} t}$ is used to transform the $\mathrm{d} t$ integration into $\mathrm{a} d s$ integration. Equation (48) means that the integral $\int_{0}^{T}(\ldots) \mathrm{d} t$ is independent of the dynamics of the spin precession and depends only on the geometry of the curve $\mathcal{S}$, thereby justifying the denotation as "geometrical part of the (classical) quasienergy.” Note, however, that $\epsilon_{g}^{(c l)}$ still depends on the period $T$ according to the prefactor $\frac{1}{T}$ in (47). The integral (48) can be identified with the geometric phase of the Bloch sphere [5, 22], but we will not dwell upon this aspect in the present article.

Furthermore, for the calculation of $\epsilon_{g}^{(c l)}$, we could choose any magnetic field $\mathbf{h}(s)$ that yields the curve $\mathcal{S}$ when solving the corresponding equation of motion.

The following choices considerably simplify the calculations: As a parameter of $\mathcal{S}$, we will use the arc length that will always be denoted by $s$ in what follows. Differentiation with respect to $s$ will again be denoted by a prime' without danger of confusion. The length of the curve $\mathcal{S}$ will be denoted by $|\mathcal{S}|$. This has the consequence that

$$
\begin{array}{r}
v \equiv\left\|\mathbf{s}^{\prime}(s)\right\|=1 \text { and } \mathbf{s}^{\prime}(s) \cdot \mathbf{s}(s)=0 \\
\text { for all } s \in[0,|\mathcal{S}|) .
\end{array}
$$

Further, we will choose as a suitable magnetic field

$$
\mathbf{h}(s)=\mathbf{s}(s) \times \mathbf{s}^{\prime}(s)
$$

which will always be of unit length,

$$
\|\mathbf{h}(s)\|=1,
$$

as the vector product of two orthogonal unit vectors. Equation (48) yields the correct spin curve $\mathcal{S}$ as it satisfies

$$
\mathbf{h} \times \mathbf{s}=\left(\mathbf{s} \times \mathbf{s}^{\prime}\right) \times \mathbf{s} \stackrel{(49)}{=} \mathbf{s}^{\prime} .
$$

As $\mathbf{h} \cdot \mathbf{s}=0$, the dynamical part $\epsilon_{d}^{(c l)}$ of the quasienergy always vanishes. For reasons that will become clear later, we call (50) the "dual magnetic field" of the spin vector function $\mathbf{s}(s)$, and the curve $\mathcal{H}$ swept by $\mathbf{h}(s)$ the "dual loop" of the loop $\mathcal{S}$. With the above choices, the geometric part of the quasienergy can be written as

$$
\epsilon_{g}^{(c l)}=\frac{1}{T} \int_{0}^{|\mathcal{S}|}\left(-\mathbf{s} \cdot\left(\mathbf{s}^{\prime} \times \mathbf{s}^{\prime \prime}\right)\right) \mathrm{d} s .
$$

It is known from elementary differential geometry that the "geodesic curvature" $k_{g}$ of a curve $\mathcal{S}$ on a surface parametrised by its arc length is defined as the triple product

$$
k_{g} \equiv \mathbf{s} \cdot\left(\mathbf{s}^{\prime} \times \mathbf{s}^{\prime \prime}\right)
$$

measuring the component of the acceleration $\mathbf{s}^{\prime \prime}$ in the tangent plane of the curve (see, e.g. [23]). It can have positive or negative values and vanishes at the inflection points of the curve. It follows that the integrand in (53) can be, up to a sign, interpreted as the geodesic curvature $k_{g}$ of $\mathcal{S}$. Inspired by [24], we will apply the theorem of Gauss-Bonnet [23] for the unit sphere that may be written as

$$
\int_{M} K \mathrm{~d} A+\int_{\partial M} k_{g} \mathrm{~d} s=2 \pi .
$$

Here $M$ denotes a two-dimensional submanifold of $S^{2}$ with boundary $\partial M$ and (constant) Gaussian curvature $K$. In our case, we set $\partial M=\mathcal{S}$ and can identify the surface integral $\int_{M} K \mathrm{~d} A$ with the (signed) solid angle $\mathcal{A}(\mathcal{S})$ encircled by the loop $\mathcal{S}$ and thus rewrite (55) in the form

$$
\int_{0}^{|\mathcal{S}|}\left(-\mathbf{s} \cdot\left(\mathbf{s}^{\prime} \times \mathbf{s}^{\prime \prime}\right)\right)=-\int_{\mathcal{S}} k_{g} \mathrm{ds} \stackrel{(55)}{=} \mathcal{A}(\mathcal{S})-2 \pi .
$$

The last term $-2 \pi$ is irrelevant as the quasienergy is only defined modulo $\omega=\frac{2 \pi}{T}$. Thus, we have reestablished the result

$$
\epsilon_{g}^{(c l)}=\frac{1}{T} \mathcal{A}(\mathcal{S}) \bmod \omega,
$$

that endows $\epsilon_{g}^{(c l)}$ with a geometric meaning in accordance with [5]. 


\section{Duality of Loops}

We start with a loop $\mathcal{S}$ and its dual loop $\mathcal{H}$ on the unit Bloch sphere parametrised by the arc length $s$ of $\mathcal{S}$ such that $\mathbf{s}(s)$ and $\mathbf{h}(s)$ satisfy

$$
\frac{\mathrm{d}}{\mathrm{d} s} \mathbf{s}=\mathbf{h} \times \mathbf{s}
$$

and

$$
\mathbf{h}=\mathbf{s} \times \frac{\mathrm{d}}{\mathrm{d} s} \mathbf{s} .
$$

(We will avoid the use of primes for derivatives in this section in order to avoid misunderstandings.) Hence, the triple $\left(\mathbf{h}, \mathbf{s}, \frac{\mathrm{d}}{\mathrm{ds}} \mathbf{s}\right)$ will be a right-handed orthonormal frame for all values of the parameter $s$. It follows that

$$
\frac{\mathrm{d}}{\mathrm{d} s} \mathbf{h} \stackrel{(59)}{=} \mathbf{s} \times \frac{\mathrm{d}^{2}}{\mathrm{~d} s^{2}} \mathbf{s}
$$

is orthogonal to $\mathbf{h}$ and $\mathbf{s}$ and hence must be proportional to $\frac{\mathrm{d}}{\mathrm{ds}} \mathbf{s}$ :

$$
\frac{\mathrm{d}}{\mathrm{d} s} \mathbf{h}(s)=a(s) \frac{\mathrm{d}}{\mathrm{d} s} \mathbf{s},
$$

where $a(s)$ is a smooth function. Moreover,

$$
\begin{gathered}
\frac{\mathrm{d}^{2}}{\mathrm{~d} s^{2}} \mathbf{s} \stackrel{(58)}{=} \frac{\mathrm{d}}{\mathrm{d} s}(\mathbf{h} \times \mathbf{s})=\left(\frac{\mathrm{d}}{\mathrm{d} s} \mathbf{h}\right) \times \mathbf{s}+\mathbf{h} \times\left(\frac{\mathrm{d}}{\mathrm{d} s} \mathbf{s}\right) \\
\stackrel{(58,61)}{=} a\left(\frac{\mathrm{d}}{\mathrm{d} s} \mathbf{s}\right) \times \mathbf{s}+\mathbf{h} \times(\mathbf{h} \times \mathbf{s}) \\
\stackrel{(59)}{=}-a \mathbf{h}-\mathbf{s},
\end{gathered}
$$

and hence

$$
g \equiv\left(\mathbf{s} \times \frac{\mathrm{d}}{\mathrm{d} s} \mathbf{s}\right) \cdot \frac{\mathrm{d}^{2}}{\mathrm{~d} s^{2}} \mathbf{s} \stackrel{(59,62)}{=}-a \mathbf{h} \cdot \mathbf{h}=-a .
$$

Now (58), (61), and (63) imply

$$
\frac{\mathrm{d}}{\mathrm{d} s} \mathbf{h}=-g \frac{\mathrm{d}}{\mathrm{d} s} \mathbf{s}=(g \mathbf{s}) \times \mathbf{h} \equiv \tilde{\mathbf{s}} \times \mathbf{h} .
$$

The latter equation has the form of (4) and hence can be interpreted in such a way that the "spin vector" $h$ moves according to (4) under the influence of the "magnetic

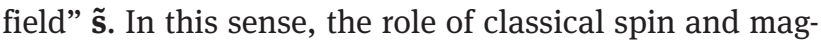
netic field is interchanged. However, in general, $\tilde{\mathbf{s}}$ will not be a unit vector, and $s$ will not be the arc length parameter of the loop $\mathcal{H}$.

The situation will be more symmetric if we additionally pass from $s$ to the arc length parameter of $\mathcal{H}$, denoted by $r$. Then, the equation of motion for $\mathbf{h}(r)$ assumes the form

$$
\frac{\mathrm{d}}{\mathrm{d} r} \mathbf{h}=\frac{\mathrm{d} s}{\mathrm{~d} r} \frac{\mathrm{d}}{\mathrm{d} s} \mathbf{h} \stackrel{(64)}{=}\left(\frac{\mathrm{d} s}{\mathrm{~d} r} g \mathbf{s}\right) \times \mathbf{h} \equiv \overline{\mathbf{s}} \times \mathbf{h} .
$$

Now the new "magnetic field" $\overline{\mathbf{s}}$ has unit length as it is the vector product of two orthogonal unit vectors:

$$
\mathbf{h} \times \frac{\mathrm{d}}{\mathrm{d} r} \mathbf{h}=\mathbf{h} \times(\overline{\mathbf{s}} \times \mathbf{h})=\overline{\mathbf{s}} \underbrace{\mathbf{h} \cdot \mathbf{h}}_{1}-\mathbf{h} \underbrace{\overline{\mathbf{s}} \cdot \mathbf{h}}_{0}=\overline{\mathbf{s}} .
$$

This means that

$$
\|\overline{\mathbf{s}}\|=\left\|\frac{\mathrm{d} s}{\mathrm{~d} r} g \mathbf{s}\right\|=\|\mathbf{s}\|=1,
$$

and hence

$$
\frac{\mathrm{d} s}{\mathrm{~d} r}=\left|\frac{1}{g}\right| .
$$

Together with (65), this implies

$$
\overline{\mathbf{s}}(r)= \pm \mathbf{s}(r) .
$$

If the role of $\mathcal{S}$ and $\mathcal{H}$ is interchanged, we obtain

$$
\frac{\mathrm{d} r}{\mathrm{~d} s}=|g|=\left|\frac{1}{G}\right|,
$$

where $G$ is the geodesic curvature of $\mathcal{H}$ :

$$
G \equiv \mathbf{h} \cdot\left(\frac{\mathrm{d} \mathbf{h}}{\mathrm{d} r} \times \frac{\mathrm{d}^{2} \mathbf{h}}{\mathrm{d} r^{2}}\right) .
$$

Using (59) and (63), we may rewrite (62) as

$$
\frac{\mathrm{d}^{2}}{\mathrm{~d} s^{2}} \mathbf{s}=-\mathbf{s}+g \mathbf{s} \times \frac{\mathrm{d}}{\mathrm{ds}} \mathbf{s} .
$$

Then, it follows that

$$
\begin{aligned}
\frac{\mathrm{d}}{\mathrm{d} s} \mathbf{h} & \stackrel{(60)}{=} \mathbf{s} \times \frac{\mathrm{d}^{2}}{\mathrm{~d} s^{2}} \mathbf{s} \\
& \stackrel{(72)}{=} \mathrm{g} \mathbf{s} \times\left(\mathbf{s} \times \frac{\mathrm{d}}{\mathrm{d} s} \mathbf{s}\right)=-\mathrm{g} \frac{\mathrm{d}}{\mathrm{d} s} \mathbf{s},
\end{aligned}
$$

and hence

$$
\left\|\frac{\mathrm{d}}{\mathrm{d} s} \mathbf{h}\right\|=|g| \text {. }
$$

This means that, up to a possible sign, the geodesic curvature differential $g$ d $s$ of $\mathcal{S}$ equals the arc length differential $\mathrm{d} r$ of $\mathcal{H}$. If $g$ does not change its sign, both differentials can be integrated and yield identical integrals, up to a possible sign. 
The curve $\mathcal{S}$ will be called simple if its geodesic curvature does not change its sign. In this case, we obtain

$$
\int_{0}^{|\mathcal{S}|} k_{g} \mathrm{~d} s= \pm|\mathcal{H}|
$$

and, by means of (56) and (57),

$$
\epsilon_{g}^{(c l)}= \pm \frac{|\mathcal{H}|}{|\mathcal{S}|} \bmod \omega
$$

The result (75) reminds of the relationship between the geometric phase and the contracted length of the system's path in projective Hilbert space according to [25].

Summarising, we have two loops $\mathcal{S}$ and $\mathcal{H}$ on the unit Bloch sphere that give rise to two different solutions of (4): Either $\mathcal{S}$ consists of spin vectors or $\mathcal{H}$ consists of magnetic field vectors, and the time parameter $t$ in (4) is chosen as the arc length $s$ of $\mathcal{S}$. Or, $\mathcal{H}$ consists of spin vectors and $\pm \mathcal{S}$ of magnetic field vectors, and the time parameter $t$ in (4) is chosen as the arc length parameter $r$ of $\mathcal{H}$. This symmetry between $\mathcal{S}$ and $\mathcal{H}$ justifies the denotation as "dual loops."

For both realisations of solutions of (4), we can calculate the classical quasienergy denoted by $\epsilon_{S}^{(c l)}$ and $\epsilon_{H}^{(c l)}$, respectively. It consists only of its geometric part as spin vector and magnetic field will be orthogonal in both realisations. For the case of simple spin curves, (76) immediately implies that there are representatives $\epsilon_{S}^{(c l)}$ and $\epsilon_{H}^{(c l)}$ such that

$$
\epsilon_{S}^{(c l)}= \pm \frac{1}{\epsilon_{H}^{(c l)}}
$$

further illustrating the duality between $\mathcal{S}$ and $\mathcal{H}$.

We note that there is still a minor asymmetry between the curves $\mathcal{S}$ and $\mathcal{H}$ of a dual pair, insofar the two branches $\pm \mathcal{S}$ occur only at the $\mathcal{S}$ side. Recall that both branches correspond to physical solutions as (4) is invariant under the reflection $\mathbf{s} \mapsto-\mathbf{s}$. The introduction of $\pm \mathcal{H}$ would mean that we consider both orientations of $\pm \mathcal{S}$ on an equal footing, as (4) is also invariant under the simultaneous reflections $\mathbf{h} \mapsto-\mathbf{h}$ and $t \mapsto-t$. Thus, the completely symmetric duality would have to be defined between $\pm \mathcal{S}$ and $\pm \mathcal{H}$.

Still another way of looking at the duality of loops considered in this section would be based on the observation that the equation of motion (4) can be viewed as a Hamiltonian equation with a two-dimensional phase space $S^{2}$ and the time-dependent Hamiltonian

$$
H(\mathbf{s}, t)=\mathbf{h}(t) \cdot \mathbf{s},
$$

see [5]. In the special case where $t$ is the arc length parameter of $\mathcal{S}$, it follows that $\mathbf{h}(t)$ can be chosen as $\mathbf{h}(t) \in S^{2}$. Due to the symmetry of the scalar product in (77), we may then swap the role of $\mathbf{h}(t)$ and $\mathbf{s}(t)$ and regard (77) as the Hamiltonian depending on the phase space variable $\mathbf{h} \in S^{2}$ and the $t$-depending field $\mathbf{s}(t)$. Any solution of the corresponding Hamiltonian equation can then be interpreted in two different ways as explained above.

\subsection{Example 1}

In order to illustrate the notion of duality considered in this section, we consider two examples. The first one is a special case of the Rabi problem with circular polarisation. Let

$$
\mathbf{s}(s)=\left(\begin{array}{c}
\sqrt{1-z^{2}} \cos \left(\frac{s}{\sqrt{1-z^{2}}}\right) \\
\sqrt{1-z^{2}} \sin \left(\frac{s}{\sqrt{1-z^{2}}}\right) \\
z
\end{array}\right),
$$

be the arc length parametrisation of a circle $\mathcal{S}$ on $S^{2}$ lying in the plane $z=$ const. with $-1<z<1$ and $z \neq 0$. This leads to

$$
|\mathcal{S}|=2 \pi \sqrt{1-z^{2}} \equiv \frac{2 \pi}{\omega} .
$$

Then, (59) yields the parametrisation of the dual loop $\mathcal{H}:$

$$
\mathbf{h}(s)=\left(\begin{array}{c}
-z \cos \left(\frac{s}{\sqrt{1-z^{2}}}\right) \\
-z \sin \left(\frac{s}{\sqrt{1-z^{2}}}\right) \\
\sqrt{1-z^{2}}
\end{array}\right),
$$

satisfying

$$
|\mathcal{H}|=2 \pi|z| \equiv \frac{2 \pi}{\Omega} .
$$

After some elementary calculations, we obtain

$$
g=\left(\mathbf{s} \times \frac{\mathrm{d}}{\mathrm{ds}} \mathbf{s}\right) \cdot \frac{\mathrm{d}^{2}}{\mathrm{~d} s^{2}} \mathbf{s}=\frac{z}{\sqrt{1-z^{2}}},
$$

and hence, the first expression for the classical quasienergy $\epsilon_{S}^{(c l)}$ reads

$$
\epsilon_{S}^{(c l 1)} \stackrel{(53)}{=} \frac{1}{|\mathcal{S}|} \int_{0}^{|\mathcal{S}|}(-g) \mathrm{d} s=-g=-\frac{z}{\sqrt{1-z^{2}}} .
$$


The solid angle enclosed by $\mathcal{S}$ will be

$$
\mathcal{A}(\mathcal{S})=2 \pi(1-z) \stackrel{(55,75)}{=} 2 \pi \pm|\mathcal{H}|,
$$

and hence, a second expression for $\epsilon_{S}^{(c l)}$ will be

$$
\epsilon_{S}^{(c l 2)} \stackrel{(57)}{=} \frac{\mathcal{A}(\mathcal{S})}{|\mathcal{S}|}=\frac{\sqrt{1-z}}{\sqrt{1+z}} .
$$

It satisfies

$$
\epsilon_{S}^{(c l 2)}=\epsilon_{S}^{(c l 1)}+\omega
$$

and hence, both expressions (84) and (86) agree modulo $\omega$.

The arc length parameter $r$ corresponding to $\mathcal{H}$ is obtained as

$$
r \stackrel{(68)}{=} g s \stackrel{(83)}{=} \frac{z}{\sqrt{1-z^{2}}} s .
$$

After some elementary calculations, we obtain

$$
G \equiv\left(\mathbf{h} \times \frac{\mathrm{d}}{\mathrm{d} r} \mathbf{h}\right) \cdot \frac{\mathrm{d}^{2}}{\mathrm{~d} r^{2}} \mathbf{h}=\frac{\sqrt{1-z^{2}}}{z} \stackrel{(83)}{=} \frac{1}{g},
$$

and hence, the first expression for the quasienergy $\epsilon_{H}^{(c l)}$ reads

$$
\epsilon_{H}^{(c l 1)} \stackrel{(53)}{=} \frac{1}{|\mathcal{H}|} \int_{0}^{|\mathcal{H}|}(-G) \mathrm{d} r=-G \stackrel{(89)}{=}-\frac{\sqrt{1-z^{2}}}{z} .
$$

The solid angle enclosed by $\mathcal{H}$ will be

$$
\mathcal{A}(\mathcal{H})=2 \pi\left(1-\sqrt{1-z^{2}}\right) \stackrel{(55,75)}{=} 2 \pi-|\mathcal{S}|,
$$

and hence, a second expression for $\epsilon_{S}^{(c l)}$ is given by

$$
\epsilon_{H}^{(c l 2)} \stackrel{(57)}{=} \frac{\mathcal{A}(\mathcal{H})}{|\mathcal{H}|}=\frac{1-\sqrt{1-z^{2}}}{|z|}
$$

It satisfies

$$
\epsilon_{H}^{(c l 2)}= \pm \epsilon_{H}^{(c l 1)}+\Omega
$$

the \pm sign depending on the sign of $z$. Hence, both expressions (90) and (93) agree up to a sign and modulo $\Omega$.

Equation (77) holds for the present example as the triple products $g$ and $G$ are constant and, due to (89), inverses of each other.

In the case of $0<z<1$, the curve $\mathcal{S}$ of our example generates a closed, convex cone $\mathcal{C}(\mathcal{S}) \subset \mathbb{R}^{3}$, analogously for the dual curve $\mathcal{H}$. Then, it follows that $\mathcal{C}(\mathcal{H})$ is the dual cone of $\mathcal{C}(\mathcal{S})$ and vice versa. Here, the dual cone $C^{\prime}$ of a cone $C$ is defined by

$$
C^{\prime} \equiv\left\{\mathbf{x} \in \mathbb{R}^{3} \mid \mathbf{x} \cdot \mathbf{y} \geq 0 \text { for all } \mathbf{y} \in C\right\},
$$

see, e.g. [26]. In this sense, our definition of dual curves is compatible with the established notion of dual cones in $\mathbb{R}^{3}$.

Finally, we note that the magnetic field (81) can be understood as a special case of the Rabi problem with circularly polarised driving (23), if we set $F=-z, \omega=\frac{1}{\sqrt{1-z^{2}}}$ and $\omega_{0}=\sqrt{1-z^{2}}$. For this problem, the quasienergy $\epsilon_{c}$ assumes the form

$$
\epsilon_{c}=\sqrt{F^{2}+\left(\omega_{0}-\omega\right)^{2}}
$$

see [5], (8) and taking into account that the quasienergy of the classical Rabi problem is twice the quasienergy of the $s=1 / 2$ quantum Rabi problem modulo $\omega$, see also Proposition 1. In our case, it follows that

$$
\epsilon_{c}=\sqrt{z^{2}+\left(\frac{1}{\sqrt{1-z^{2}}}-\sqrt{1-z^{2}}\right)^{2}}=\frac{|z|}{\sqrt{1-z^{2}}},
$$

which agrees with (84) up to a possible sign.

\subsection{Example 2}

For the second example, we take a case where $\mathcal{S}$ is not simple, but of the form of the figure " 8 " (lemniscate) with a self-intersection that is simultaneously a point of inflection. This example also shows that we need not explicitly calculate the arc length parameters $s$ of $\mathcal{S}$ or $r$ of $\mathcal{H}$ but may work within the initial parametrisation. Let the figure "8" curve be given by the parametrisation

$$
\mathbf{s}(\tau)=\frac{1}{\sqrt{\sin ^{2}(\tau)+\sin ^{2}(2 \tau)+1}}\left(\begin{array}{c}
\sin \tau \\
\sin 2 \tau \\
1
\end{array}\right),
$$

where $\tau \in[0,2 \pi]$. We calculate the dual loop $\mathcal{H} \in S^{2}$ by

$$
\mathbf{h}(\tau)=\frac{1}{\left\|\frac{\mathrm{d} \mathbf{s}}{\mathrm{d} \tau}\right\|} \mathbf{s} \times \frac{\mathrm{d} \mathbf{s}}{\mathrm{d} \tau},
$$

analogously to (59) but without directly using the arc length parameter $s$. This and the following expressions can be easily obtained by a computer algebra software but are too involved to be displayed here. The loop $\mathcal{H}$ is displayed in Figure 2. It shows two cusps corresponding to 


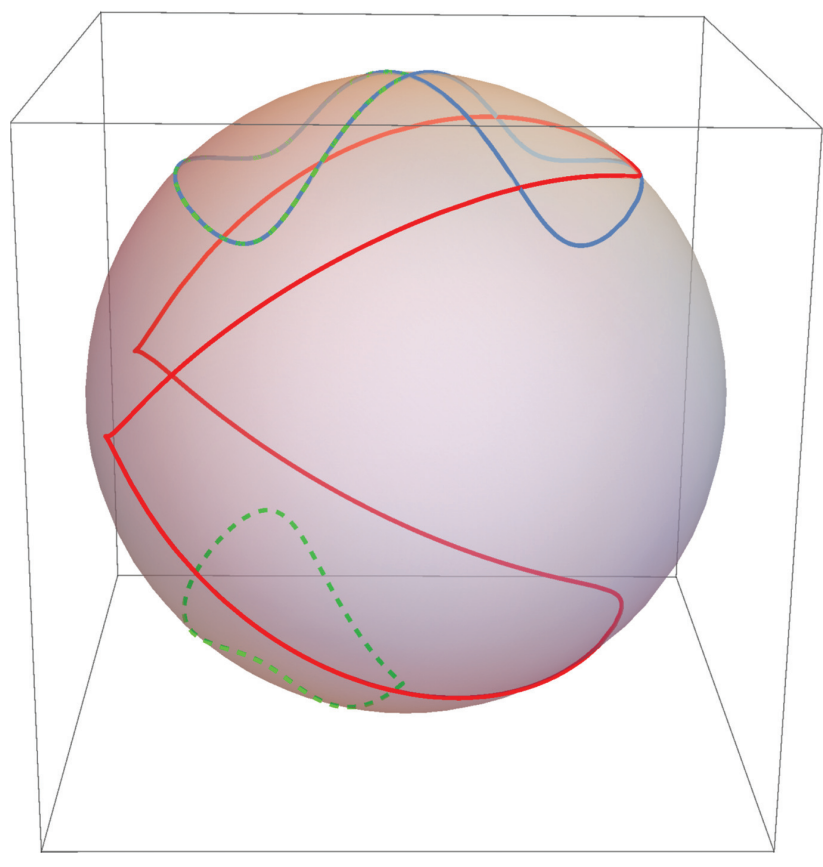

Figure 2: Illustration of example 2 for duality of loops. The blue (grey) curve represents the orbit $\mathcal{S}$ of a time-dependent spin function $\mathbf{s}(\tau)$ according to (97); the red (dark) one is the dual loop of $\mathcal{H}$ of magnetic field vectors. If we iterate the construction we obtain for the bidual the two green dashed curves $\mathcal{R}$ that locally coincide with $\mathcal{S}$ or $-\mathcal{S}$.

the point of self-intersection of $\mathcal{S}$. These cusps necessarily occur according to the following reasoning: At the point of self-intersection corresponding to the values $\tau=0, \pi$ of the parameter, the geodesic curvature $g$ of $\mathcal{S}$ changes its sign (Fig. 3). According to (69), the geodesic curvature $G$ of $\mathcal{H}$ must diverge at $\tau=0, \pi$, which explains the two cusps.

The curve $\mathcal{S}$ can be divided into the parts $\mathcal{S}_{1}$ and $\mathcal{S}_{2}$ that have in common only the point of self-intersection. The corresponding parts of $\mathcal{H}$ are denoted by $\mathcal{H}_{i}, i=1,2$. Both parts $\mathcal{S}_{i}$ encircle solid angles $\mathcal{A}\left(\mathcal{S}_{i}\right) \approx \pm 4.64172$ that

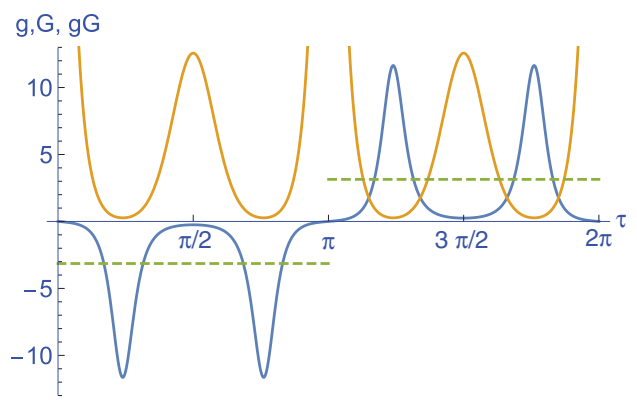

Figure 3: Plot of the geodesic curvature $g$ of $\mathcal{S}$ (blue/dark curve), $G$ of $\mathcal{H}$ (orange/grey curve), and the product $g G$ (green dashed lines) as a function of $\tau$. The data are the same as for Figure 2. According to (70), the product $g G$ must have the absolute value 1 . correspond to the length of the $\mathcal{H}_{i}$. But due to the different signs, the total solid angle and the corresponding quasienergy vanish.

Interestingly, if we calculate the "bidual" loop $\mathcal{R}$ according to

$$
r(\tau)=\frac{1}{\left\|\frac{\mathrm{dh}}{\mathrm{d} \tau}\right\|} \mathbf{h} \times \frac{\mathrm{d} \mathbf{h}}{\mathrm{d} \tau},
$$

then, we obtain two disjoint curves that locally coincide with $-\mathcal{S}_{1}$ and $\mathcal{S}_{2}$ (Fig. 2), again illustrating that the duality should be properly defined between $\pm \mathcal{S}$ and $\pm \mathcal{H}$.

\section{Summary and Outlook}

We have considered some geometrical aspects of the classical Rabi problem with arbitrary periodic driving. The classical equation of motion can be viewed in its own right as a case where Floquet theory can be applied, without resort to the underlying Schrödinger equation. In contrast to the latter, it has always some periodic solutions. This leads to the definition of the classical quasienergy $\epsilon^{(c l)}$ that has an integral representation in terms of the periodic solution and possesses a natural splitting into a dynamical part $\epsilon_{d}^{(c l)}$ and a geometrical one $\epsilon_{g}^{(c l)}$. The latter is essentially a geometrical phase on the Bloch sphere and can be related to the solid angle swept by the classical spin during one period via the theorem of Gauss-Bonnet. If we focus on the loops $\pm \mathcal{S}$ swept by periodic solutions of the classical Rabi problem without fixing its parametrisation by the time parameter $t$, we may assume that $\pm \mathcal{S}$ is produced by a pair of loops $\pm \mathcal{H}$ representing time-dependent magnetic fields of unit length. Upon using the respective arc length parameters, the role of spin and magnetic field can be interchanged. We have further analysed this duality between $\pm \mathcal{S}$ and $\pm \mathcal{H}$ that in the case of curves $\pm \mathcal{S}$ without points of inflection even leads to pairs of reciprocal quasienergies.

It is not straightforward to assess the possible benefits of the present results for concrete physical problems, also because of the diversity of such problems. However, as a rule, the solutions of the equations of motion of the classical spin can be more directly visualised than the solutions of the corresponding Schrödinger equation. Although this advantage is lost in higher-dimensional Hilbert spaces, it would be instructive to investigate which geometric properties remain invariant if we pass from the Bloch sphere to a higher-dimensional projective Hilbert space. The latter is known to be a Kähler manifold that carries two related structures, a Riemannian and a symplectic one, which 
can be used to obtain geometric phases in different ways $[27,28]$.

Acknowledgements: I thank the members of the DFG Research Unit FOR 2692 for stimulating and insightful discussions on the topic of this article. I am also grateful for valuable suggestions from some anonymous referees and their references to relevant literature.

\section{Appendix A: Proof of the Integral Representation of the Classical Quasienergy}

We use the abbreviation

$$
v(t)^{2} \equiv \dot{\mathbf{s}}(t) \cdot \dot{\mathbf{s}}(t) \quad \Rightarrow \quad v \dot{v}=\dot{\mathbf{s}} \cdot \ddot{\mathbf{s}},
$$

and expand the magnetic field with respect to the e frame, defined in (38-40):

$$
\mathbf{h}(t)=\sum_{i=1}^{3} k_{i}(t) \mathbf{e}^{(i)}(t) .
$$

The equation

$$
\begin{aligned}
\dot{\mathbf{s}} & \stackrel{(39)}{=} v \mathbf{e}^{(2)} \\
& \stackrel{(4)}{=} \mathbf{h} \times \mathbf{s}=\left(k_{1} \mathbf{e}^{(1)}+k_{2} \mathbf{e}^{(2)}+k_{3} \mathbf{e}^{(3)}\right) \times \mathbf{e}^{(1)} \\
& =-k_{2} \mathbf{e}^{(3)}+k_{3} \mathbf{e}^{(2)}
\end{aligned}
$$

immediately implies

$$
k_{2}=0, \quad k_{3}=v,
$$

and moreover,

$$
k_{1}=\mathbf{h} \cdot \mathbf{s} \text {. }
$$

We will also expand $\ddot{\mathbf{s}}$ with respect to the e frame. First, we obtain

$$
\dot{\mathbf{s}} \cdot \mathbf{s}=0 \Rightarrow \dot{\mathbf{s}} \cdot \dot{\mathbf{s}}+\ddot{\mathbf{s}} \cdot \mathbf{s}=0 \Rightarrow \ddot{\mathbf{s}} \cdot \mathbf{s}=-v^{2}
$$

Second,

$$
\begin{aligned}
\ddot{\mathbf{s}} \cdot \mathbf{e}^{(3)} & =\ddot{\mathbf{s}} \cdot\left(\mathbf{e}^{(1)} \times \mathbf{e}^{(2)}\right)=\ddot{\mathbf{s}} \cdot\left(\mathbf{s} \times \frac{1}{v} \dot{\mathbf{s}}\right) \\
& =\frac{1}{v} \mathbf{s} \cdot(\dot{\mathbf{s}} \times \ddot{\mathbf{s}}) \equiv \frac{g}{v},
\end{aligned}
$$

where we have abbreviated the triple product $\mathbf{s} \cdot(\dot{\mathbf{s}} \times \ddot{\mathbf{s}})$ by g. Together with (100), the last two equations yield

$$
\ddot{\mathbf{s}}=-v^{2} \mathbf{e}^{(1)}+\dot{v} \mathbf{e}^{(2)}+\frac{g}{v} \mathbf{e}^{(3)} .
$$

With this, we can evaluate the $t$ derivatives of the $\mathbf{e}$ frame vectors in the following way:

$$
\begin{aligned}
& \dot{\mathbf{e}}^{(2)}=\frac{\mathrm{d}}{\mathrm{d} t} \frac{\dot{\mathbf{s}}}{v}=-\frac{\dot{v}}{v^{2}} \dot{\mathbf{s}}+\frac{1}{v} \ddot{\mathbf{s}} \stackrel{(107)}{=}-v \mathbf{e}^{(1)}+\frac{g}{v^{2}} \mathbf{e}^{(3)}, \\
& \dot{\mathbf{e}}^{(3)} \stackrel{(40)}{=} \dot{\mathbf{e}}^{(1)} \times \mathbf{e}^{(2)}+\mathbf{e}^{(1)} \times \dot{\mathbf{e}}^{(2)} \\
&=\frac{1}{v} \underbrace{\dot{\mathbf{s}} \times \dot{\mathbf{s}}}_{0}+\frac{g}{v^{2}} \mathbf{e}^{(1)} \times \mathbf{e}^{(3)} \\
&=-\frac{g}{v^{2}} \mathbf{e}^{(2)} .
\end{aligned}
$$

The $t$ derivative of $\mathbf{s}^{(2)}(t)$ can now be calculated in two different ways:

$$
\begin{aligned}
\dot{\mathbf{s}}^{(2)} \stackrel{(4)}{=} & \mathbf{h} \times \mathbf{s}^{(2)} \\
= & \left((\mathbf{h} \cdot \mathbf{s}) \mathbf{e}^{(1)}+v \mathbf{e}^{(3)}\right) \\
& \times\left(\cos \alpha \mathbf{e}^{(2)}+\sin \alpha \mathbf{e}^{(3)}\right) \\
= & -v \cos \alpha \mathbf{e}^{(1)}-(\mathbf{h} \cdot \mathbf{s}) \sin \alpha \mathbf{e}^{(2)} \\
& +(\mathbf{h} \cdot \mathbf{s}) \sin \alpha \mathbf{e}^{(3)},
\end{aligned}
$$

and

$$
\begin{aligned}
\dot{\mathbf{s}}^{(2)} \stackrel{(44)}{=} & -\dot{\alpha} \sin \alpha \mathbf{e}^{(2)}+\cos \alpha \dot{\mathbf{e}}^{(2)} \\
& +\dot{\alpha} \cos \alpha \mathbf{e}^{(3)}+\sin \alpha \dot{\mathbf{e}}^{(3)} \\
= & \dot{\alpha}\left(-\sin \alpha \mathbf{e}^{(2)}+\cos \alpha \mathbf{e}^{(3)}\right) \\
& +\cos \alpha\left(-v \mathbf{e}^{(1)}+\frac{g}{v^{2}} \mathbf{e}^{(3)}\right) \\
& -\sin \alpha \frac{g}{v^{2}} \mathbf{e}^{(2)} .
\end{aligned}
$$

Comparing the $\mathbf{e}^{(2)}$ components of (110) and (111) yields the expression for $\dot{\alpha}$ we are looking for:

$$
\dot{\alpha}=(\mathbf{h} \cdot \mathbf{s})-\frac{g}{v^{2}}=(\mathbf{h} \cdot \mathbf{s})-\frac{\mathbf{s} \cdot(\dot{\mathbf{s}} \times \ddot{\mathbf{s}})}{\dot{\mathbf{s}} \cdot \dot{\mathbf{s}}} .
$$

This shows that $\alpha(t)$ can be obtained as an integral over the right-hand side of (112) that is a function of $\mathbf{s}(t)$ and its first two derivatives:

$$
\alpha(t)=\int_{0}^{t}\left((\mathbf{h} \cdot \mathbf{s})-\frac{\mathbf{s} \cdot(\dot{\mathbf{s}} \times \ddot{\mathbf{s}})}{\dot{\mathbf{s}} \cdot \dot{\mathbf{s}}}\right) \mathrm{d} t^{\prime},
$$


using that $\alpha(0)=0$ according to the initial conditions (42). From this, (46) follows immediately.

Note that we have not used the fact that $\mathbf{s}(t)$ would be $T$-periodic. These calculations also show that if one solution $\mathbf{s}(t)$ of (4) is given, then the other two solutions with orthogonal initial conditions can be obtained by means of certain integrals.

\section{References}

[1] I. I. Rabi, Phys. Rev. 51, 652 (1937).

[2] J. H. Shirley, Phys. Rev. 138, 979 (1965).

[3] A. G. Rojo and A. M. Bloch, Am. J. Phys. 78, 1014 (2010).

[4] H. Kaur, S. R. Jain, and S. S. Malik, Phys. Lett. A 378, 388 (2014).

[5] H.-J. Schmidt, Z. Naturforsch. A 73, 705 (2018).

[6] H.-J. Schmidt, arXiv:1910.02444 [physics.class-ph] (2019).

[7] C. Cafaro and P. M. Alsing, Int. J. Quantum. Inf. 17, 1950025 (2019).

[8] E. Majorana, Nuovo. Cim. 9, 43 (1932).

[9] H. M. Bharath, J. Math. Phys. 59, 062105 (2018).

[10] T. Ma and S.-M. Li, arXiv:0711.1458v2 [cond-mat.other] (2007).

[11] Q. Xie and W. Hai, Phys. Rev. A 82, 032117 (2010).

[12] Q. Xie, Pramana J. Phys. 91, 19 (2018).
[13] H.-J. Schmidt, J. Schnack, and M. Holthaus, Appl. Anal. 98 (2019). doi: 10.1080/00036811.2019.1632439.

[14] M. V. Berry, Proc. R. Soc. Lond. A 329, 45 (1984).

[15] Y. Aharonov and J. Anandan, Phys. Rev. Lett. 58, 1593 (1987).

[16] K. Nagata, K. Kuramitani, Y. Sekiguchi, and H. Kosaka, Nat. Commun. 9, 3227 (2018).

[17] F. Leroux, K. Pandey, R. Rehbi, F. Chevy, C. Miniatura, et al., Nat. Commun. 9, 3580 (2018).

[18] H. M. Bharat, M. Boguslawski, M. Barrios, L. Xin, and M. S. Chapman, Phys. Rev. Lett. 123, 173202 (2019).

[19] Z. Chen, J. D. Murphree, and N. P. Bigelow, Phys. Rev. A 101, 013606 (2020).

[20] G. Floquet, Ann. Sci. Ecole. Norm. S. 12, 47 (1883).

[21] V. A. Yakubovich and V. M. Starzhinskii, Linear Differential Equations with Periodic Coefficients, 2 volumes, Wiley, New York 1975.

[22] I. Menda, N. Burič, D. B. Popovič, S. Prvanovič, and M. Radonjič, Acta Phys. Pol. A 126, 670 (2014).

[23] R. S. Millman and G. Parker, Elements of Differential Geometry, Prentice-Hall, Englewood Cliffs, NJ 1977.

[24] J. von Bergmann and H. von Bergmann, Am. J. Phys. 75, 888 (2007).

[25] A. K. Pati, Phys. Lett. A 159, 105 (1991).

[26] R. T. Rockafellar, Convex Analysis (Reprint of the 1979 Princeton Mathematical Series 28 ed.), Princeton University Press, Princeton, NJ 1997.

[27] D. N. Page, Phys. Rev. A 36, 3479 (1987).

[28] A. Uhlmann, Rep. Math. Phys. 36, 461 (1995). 